\title{
Genomic exaptation enables Lasius niger adaptation to urban environments
}

Evgenii A. Konorov ${ }^{1}$, Mikhail A. Nikitinn ${ }^{2,3}$, Kirill V Mikhailov' ${ }^{2,3}$, Sergey N. Lysenkov', Mikhail Belenky ${ }^{1}$, Peter L. Chang ${ }^{4}$, Sergey V. Nuzhdin ${ }^{4,5}$ and Victoria A. Scobeyeva ${ }^{{ }^{*}}$

From The International Conference on Bioinformatics of Genome Regulation and StructurelSystems Biology (BGRSISB-2016)

Novosibirsk, Russia. 29 August-2 September 2016

\begin{abstract}
Background: The world is rapidly urbanizing, and only a subset of species are able to succeed in stressful city environments. Efficient genome-enabled stress response appears to be a likely prerequisite for urban adaptation. Despite the important role ants play in the ecosytem, only the genomes of $\sim 13$ have been sequenced so far. Here, we present the draft genome assembly of the black garden ant Lasius niger - the most successful urban inhabitant of all ants - and we compare it with the genomes of other ant species, including the closely related Camponotus floridanus.

Results: Sequences from 272 M Illumina reads were assembled into 41,406 contigs with total length of 245 MB, and N50 of 16,382 bp, similar to other ant genome assemblies enabling comparative genomic analysis. Remarkably, the predicted proteome of $L$. niger is significantly enriched relative to other ant genomes in terms of abundance of domains involved in nucleic acid binding, DNA repair, and nucleotidyl transferase activity, reflecting transposable element proliferation and a likely genomic response. With respect to environmental stress, we note a proliferation of various detoxification genes, including glutatione-S-transferases and those in the cytochrome P450 families. Notably, the CYP9 family is highly expanded with 19 complete and 21 nearly complete members - over twice as many compared to other ants. This family exhibits the signatures of strong directional selection, with eleven positively selected positions in ligand-binding pockets of enzymes. Gene family contraction was detected for several components of the olfactory system, accompanied by instances of both directional selection and relaxation.

Conclusions: Our results suggest that the success of L. niger in urbanized areas may be the result of fortuitous coincidence of several factors, including the expansion of the CYP9 cytochrome family due to coevolution with parasitic fungi, the diversification of DNA repair systems as an answer to proliferation of retroelements, and the reduction of olfactory system and behavioral preadaptations from non-territorial subdominant life strategies found in natural environments. Diversification of cytochromes and DNA repair systems along with reduced odorant communication are the basis of L. niger pollutant resistance and polyphagy, while non-territorial and mobilization strategies allows more efficient exploitation of large but patchy food sources.
\end{abstract}

Keywords: Lasius niger, Urban environment, Draft genome, Transposable element, Detoxification, Directional selection

\footnotetext{
* Correspondence: skobei-khanum@yandex.ru

${ }^{1}$ Faculty of Biology, Department of Evolution, Lomonosov Moscow State

University, Moscow, Russian Federation

Full list of author information is available at the end of the article
} 


\section{Background}

Ants are a successful and diverse group of insects, playing a role in various ecosystem functions. These roles include facilitating soil aeration, preying on herbivore insects, farming aphids for their honeydew, and cutting leaves to farm fungi for foraging. Ant eusociality is of great scientific interest and numerous works have addressed issues in its division of labour and cast-specific gene expression. Most ant genomes were sequenced due to their status as an invasive species (Solenopsis invicta [1], Linepithema humile [2] and Cerapachys biroi [3]), for their unusual cast division of labour (C. biroi and Harpegnatos saltator [4]), or for their negative impact on the economy (Camponotus floridanus [4]).

The black garden ant Lasius niger is a usual ant of the northern hemisphere and dwells in nearly all landscapes in Europe, ranging from forests to big cities. The last review of L. niger was in [5], where the new species Lasius platythorax was established. Workers in L. niger are small (ca. $5 \mathrm{~mm}$ ), while queens can reach $16 \mathrm{~mm}$. Colonies are monogynous and have only one egg-producing queen, though polyandry does occurs [6, 7]. L. niger nests are usually underground or in sand mounds, but are also found in wall cracks and rotting wood. $L$. niger feeds primarily on honeydew and other sugarrich substances; small invertebrates are also part of their diet. Farming aphids for honeydew often occur in gardens, thus effectively making itself a pest. These ants are also known to infest human housing, causing noticeable disturbances [7]. L. niger dwells in Holarctic region, inhabiting all of Europe and parts of Asia and North America. L. niger is the most widespread of all sequenced ants.

In natural environments Lasius niger occupy a subdominant position in ant communities, while species of the genera Myrmica and Formica play the main role as generalist predators. When forest ecosystems are eroded, however, dominant ant species are the first to disappear, creating possible niches and opportunities for other ant species. Lasius niger has a very flexible ecological strategy and can inhabit different spatial tiers from soil to trees. Due to its ecological plasticity Lasius niger has grown widespread in urbanised areas: the black garden ant had even increased its abundance in highly affected territories [8].

Here we report the draft genome of $L$. niger as a means of studying the genetic mechanisms underlying insect adaptation to urban environments. We focus on gene families that could facilitate behavioural or physiological means of coping with specific urban stresses.

\section{Methods}

\section{Source material}

Population samples for sequencing were obtained from two locations: near the campus of Moscow State University and from the Istra district ca. $70 \mathrm{~km}$ from Moscow. In early September 2011 worker ants from three sites (five nests from each site) at both locations were collected using aspirators and stored separately in 98\% ethanol. Approximately 50-60 ants from each of six sites were treated as different samples. For exact sample sizes see Additional file 1: Table S1. Samples were dry-freezed and transported to the University of Southern California.

\section{Genomic procedures}

Genomic DNA for each of the six samples were prepared into six libraries (one per site) and sequenced as paired-ended $100 \mathrm{bp}$ reads on an Illumina HiSeq 2500. Each library was individually barcoded and sequenced as a pooled sample of barcoded DNA, generating 423 million (M) total reads.

Since de novo assembly of complete genomes can be sensitive to sequencing errors, it is critical that the reads used to generate contigs have the highest sequencing quality. Reads were removed from consideration in the de novo assemblyif the read had a terminal "phred" [9] (quality value less than 15), or if the read contained more than two unknown nucleotides. Reads were also filtered out with Trimmomatic [10] if they had similarity to known polymerase chain reaction primer and Illumina adapter sequences.

The $272 \mathrm{M}$ retained reads from all samples were assembled with SPAdes version 3.0.0 [11]. The data are deposited to Bioproject PRJNA171386.

Genome assembly completeness was assessed using CEGMA [12] and BUSCO [13]. CEGMA score was $98,8 \%$ compared to $98,0-99,6 \%$ range for other ant genomes [3]. The raw Illumina data and the draft assembly have been deposited in NCBI under the Bioproject PRJNA171386 and will be released upon publication.

\section{Gene prediction and Gene Ontology (GO) content}

For gene prediction AUGUSTUS [14] was used with the Nasonia vitripennis genome as a training set. The prediction was done on both strands, including partial genes. Predicted genes were searched against the NR protein database (BLASTP, max_target_seqs 20). Contigs with bacterial and fungal genes were removed from the assembly. Bacterial 16 s RNA were identified using BLAST searches against the bacterial $16 \mathrm{~s}$ RNA database; selected sequences were then searched against the NR database for genus and species identification.

For more accurate searches of genes encoding cytochromes, odorant receptors, odorant-binding proteins and desaturases, hymenopterans protein sequences were used to build alignments using hmm model with hmmbuild. We built separate models for each major CYP superfamily (CYP4, CYP6, CYP9). Hmmsearch output was checked by $\operatorname{BLAST}(\mathrm{nr})$ and delta-blast for the presence of specific 
domains. Genes with hmmersearch hits were aligned using BLASTP against the NR database, and genes with the majority of hits from other families were removed from further analysis. We used delta blast to check for gene-specific domains. We also searched for sequences from other examined species in the Hymenoptera Genomes Portal (for C. floridanus, C. obscuritor, H.saltator, A. echinatior, A. cephalotes, S. invicta, P. barbatus, L. humile) and in Genbank (for C. biroi, M .pharaonis, V. emeryi, W. auropucntata). Predicted proteins of $L$. niger and other ant species were then annotated using BLASTP (cutoff 5e-2) and BLAST2GO [15]. We performed GO mapping, BLAST-based annotation, Interproscan annotation and joined BLAST and Interproscan annotation data. GO terms were assigned for each protein, GO category numbers for each species were normalized by total protein number. Dixon Q-test for outliers was used to detect overrepresented and underrepresented GO groups in the $L$. niger genome, assuming it as an outlier among all ants. GO distribution was normalized with arcsine transformation [16] L. niger was treated as outlier in both cases - in over and under represented GO IDs. Proteins and genes of species with draft genomes that were not published yet $(C$. obscuritor, V. emeryi, W. auropunctata, M. pharaonis) were not included in GO-mapping and Intersproscan annotation and was used only for OR and CYP comparison and selection detection.

\section{Alignment and detection of selection}

To eliminate potential pseudogenes, any sequence with predicted start and stop codons was discarded if it was shorter than its hypothetical product length by $25 \%$. We assumed 300 amino acid residues for desaturases and 500 for odorant receptors and P450 cytochromes, based on rounded off mean length of respective proteins in databases. For P450 we kept only sequences containing Phe-X-X-Gly-X-Arg-X-Cys-X-Gly motif, which is believed to be crucial to stabilizing the iron ion in the heme group. The selected sequence predictions for P450, odorant receptors and desaturases were than aligned for each family using the MUSCLE software (default settings, codon alignment). For the P450 cytochrome superfamily we aligned sequences in CYP6, CYP4 and CYP9 separately for each family.

Search for best substitution models were performed in MEGA6.0. Tamura-Nei substitution modelwas found best for our dataset and was used for phylogenetic analysis. A maximum-likelihood phylogenetic tree was generated for each alignment using MEGA6.0 (gaps/missing data treatment - partial deletion, 95\%,). To identify genes under selection, we applied a Z-test for selection [17] to nucleotide alignments of these genes. The positive selection hypothesis was rejected in none of the cases. A sequence was tagged as being under positive selection if both the neutrality and the purifying selection hypotheses were rejected.

For the CYP9 subfamily of P450 cytochromes we also searched for sites under selection. For this analysis we used only complete coding sequences with start and stop codons in the length range of 1425-1800 nucleotides due to limitations of selection search methods (see below). Upon removal of stop codons, sequences were codonaligned using ClustalW software. Maximum-likelihood phylogenetic trees were then generated using MEGA6.0 (gaps/missing data treatment - complete, Tamura-Nei model, bootstrap replication number -500 , initial tree for ML - Neighboor Joining). Based on these trees, a selection search with FUBAR $(p<0.05)$ and MEME $(p<$ 0.05 ) methods was performed for each codon in a given alignment using HyPhy2.0 software. The number of amino acid substitution events were then calculated based upon ancestor sequence reconstructed with SLAC.

\section{Results}

\section{Genome assembly and annotation}

We sequenced and annotated the draft genome of Lasius niger and compared it with the draft genomes of other ants. The $272 \mathrm{M}$ reads were assembled with SPAdes [11] into 41,406 contigs of more than $500 \mathrm{bp}$ in length with a total length $245 \mathrm{MB}$ and an N50 of 16,382 bp. These figures are similar to other available ant draft genomes and enable comparative genomic analysis $[1,4]$. The assembly also included 30,191 contigs of more than $1000 \mathrm{bp}$ in length with a total length $237 \mathrm{MB}$. CG content was found to be $38 \%$, similar to that found in Camponotus floridanus (34\%) [4]. Median kmer coverage is 19, exhibiting a bimodal distribution of kmer coverage with peaks at 1 and 46.CpG dinucleotides are overrepresented in the $L$. niger genome: observed/expected CpG ratio is 1.55 , similar to other ants (12):

We identified transposable elements throughout the $\mathrm{L}$. niger genome using RepeatMasker 4.0.6 [18] against the Repbase TE library v2016-08-29 [19]. We found 3.47 MB of known transposable elements (compared to $8.6 \mathrm{MB}$ in $C$. floridanus and $30 \mathrm{MB}$ in $\mathrm{H}$. saltator using same method). However, there are many intact ORFs of transposon proteins compared to other ants (see «GO distribution» below), which suggest high recent transposon activity in the L.niger lineage. Transposons detected by RepeatMasker include 531 LINEs, mostly from R1/LOA/Jockey family, and 2858 LTR elements (1513 Gypsy/DIRS1, 755 BEL/Pao and 590 Ty1/Copia).

Application of the gene prediction tool Augustus [14] revealed 19,989 genes in L. niger contigs, with 15,350 having both start and stop codons. Annotated genes were shorter than in other ant species and contained fewer exons, but total exon number and length is similar to values observed in other ants (Additional file 1: Table S13). 
Therefore we conclude that the gene space of $L$. niger annotation is as complete as other ants, although the genome is more fragmented.

To distinguish between genes in the ant genome and those originating from microbes, we used blast search against the non-redundant protein database. Analysis of $16 \mathrm{~S}$ and 18S ribosomal RNA genes revealed the following non-ant species: 8 bacteria (Serratia marcescens, Luteibacter sp., Gluconobacter sp., Spiroplasma sp, Acetobacter sicerae, Georgenia sp., Tsukamurella paurometabola), one enthomopathogenic fungus Ophiocordyceps unilateralis, an unidentified spider and a plant from family Fabiaceae. High protein blast-hit similarity additionally identified Enterobacteriacea (Serratia + Yersinia/Klebsiella), Propionobacter, nematode, Lactobacillus, actinomycete (probable Strepomyces), and, potentially, Fusarium (see Additional file 1: Table S2). After removing hits to bacterial and fungal contigs we obtained a total of 18,247 ant genes; 12,207 potentially complete and 6040 partial sequences. All these contigs were also removed from the BioProject.

Among non-bacterial annotated contigs the vast majority (37\%) are annotated as proteins, common with Camponotus floridanus (Fig. 1). C. floridanus belongs to the same family Formicinae as L. niger and their similarity in proteins corresponds to morphological likeness. Three other ant species from the Fig. 1 Solenopsis invicta, Acromyrmex echinator and Harpegnatos saltator are highly specialized and diverged from Formicinae lineage in late Cretaceous [20].

\section{GO distribution}

The predicted proteome of L. niger is significantly $(p<0,05)$ different from other ant genomes in terms of abundance of several GO categories. The proteome is enriched in domains involved in nucleic acid binding (GO:0003676), DNA repair (GO:0006281), nucleotidyl transferase activity (GO:0016779), heterocyclic compound binding (GO:1901363) and contain fewer domains involved in neuropeptide signaling (GO:0007218). L. niger might also contain fewer odorant binding domains than other ants (77 versus 114-264), but this difference was not statistically significant in a Q-test.

Overrepresentation of nucleic acid binding domains is due to high abundance of retrotransposon genes coding gag-pol polyproteins or separate reverse transcriptases. We detected 475 pol and gag-pol genes in the L. niger genome compared to 55 in Camponotus and between 5 and 42 in other ant species. No env genes were found to match the abundance of pol and gag-pol. We conclude that the pol and gag-pol genes belong to retrotransposomes and are not from ongoing retroviral infections.

DNA repair domains are overrepresented in $L$. niger mostly because of duplications of PIF1-like helicases. The L. niger genome encodes 15 PIF1-like helicases compared to 3 in Camponotus and 1 in most other ants. Other DNA repair proteins duplicated in L. niger include chromosome structure maintenance proteins $1 \mathrm{~A}$ and 2, DNA damagebinding protein 1 and tyrosyl-phosphodiesterase.

\section{Detoxification system genes}

As our focus is on potential genomic features enabling success in urban environments, we paid special attention to the gene families involved in detoxification. GlutathioneS-transferases and Cytochrome P450 families are the most common detoxification genes among hymenoptera [21] and we examined these two families in detail. We expected to find multiple duplications in these families, and in glutathione-S-transferase family we found 15 genes, (Additional file 1: Table S3), though most had incomplete and short sequences. We found no evidence of species-specific duplications of glutathione-S-transferase in the $L$. niger genome.

We did find CYP genes being highly duplicated in $L$. niger, with 72 sequences matching CYP genes across 11

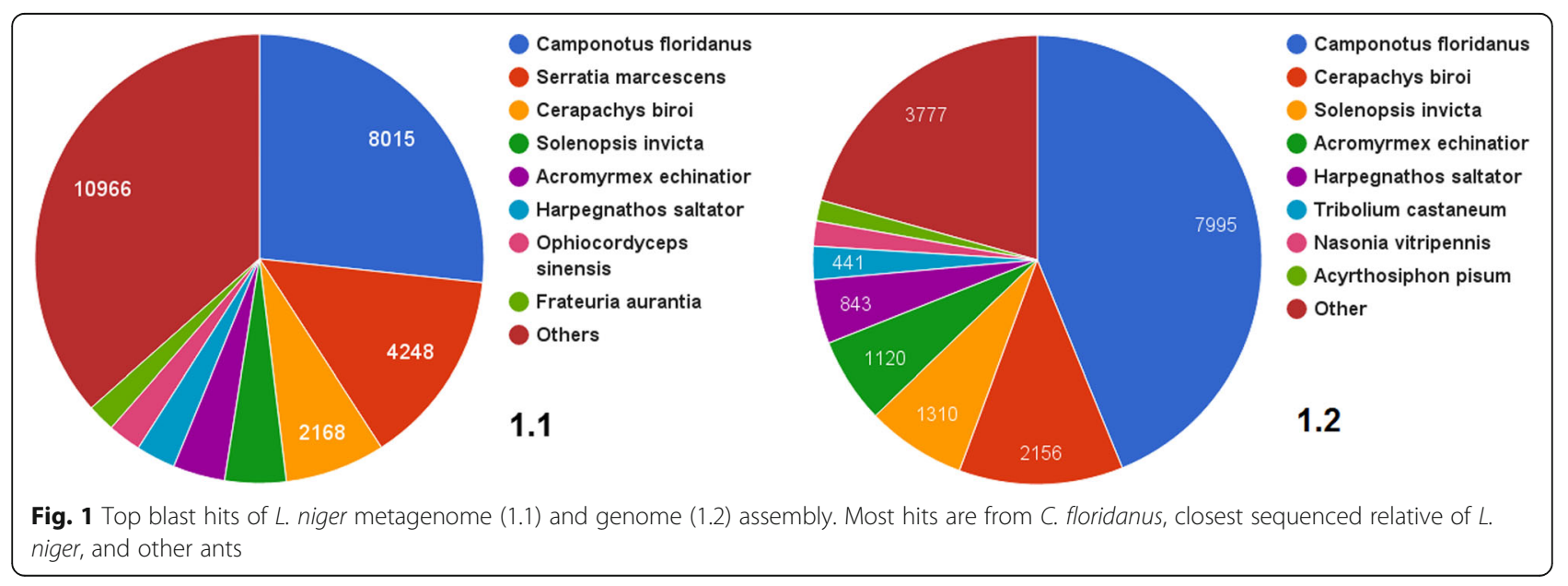


families (only complete and mostly complete genes with $>250$ aa CDS were analyzed). This is the second largest value among ants, second only to 76 CYP450 genes in C. floridanus from the same family (Additional file 1: Table S4). Compared to A. mellifera we see fewer CYP families, but more genes per family in those present (only 40 CYP450 genes found in Apis). Interestingly, there are 15 tandem clusters of CYP450 genes found in the $L$. niger genome assembly, most of which are members of CYP9E subfamily.

Most CYP450 genes in ant genomes are members of cytochrome P450 9th, 6th and 4th families (Fig. 2). The CYP9 family is highly expanded in L. niger, with 19 complete and 21 nearly complete members - over twice

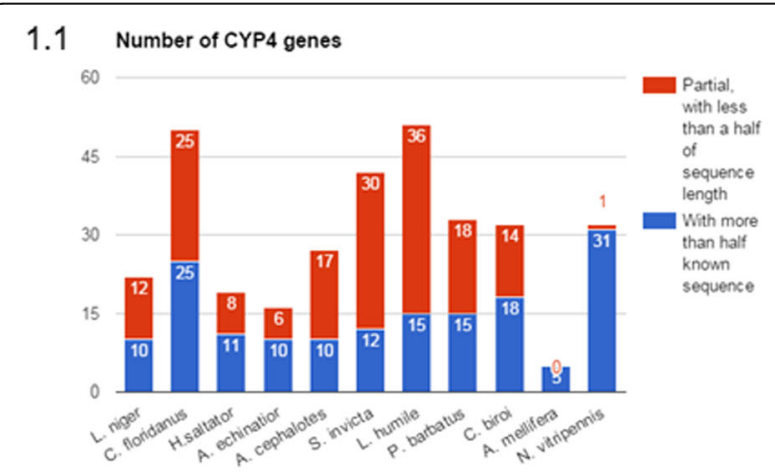

1.2 Number of CYP6 genes

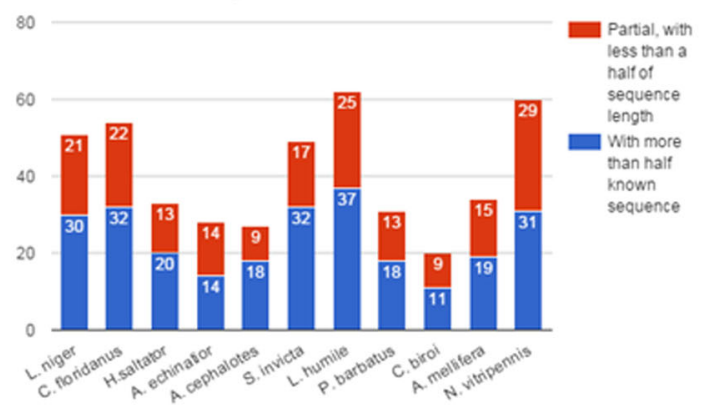

1.3 Number of CYP9 genes

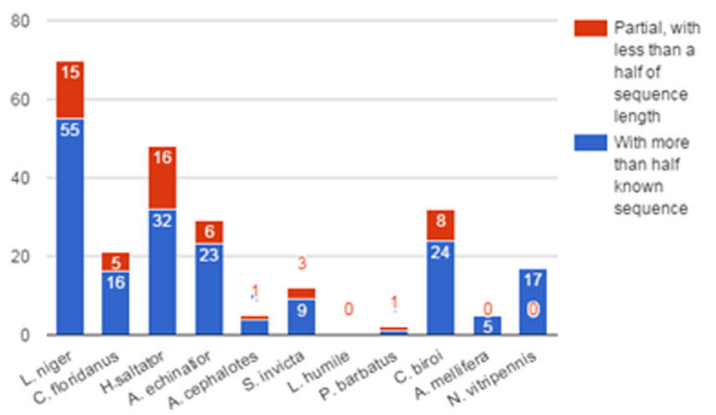

Fig. 2 Comparative abundance of CYP450 subfamilies in hymenopteran genomes. Various subfamilies are expanded in different species. Even closely related $C$. floridanus and $L$. niger differ significantly in relative abundance of CYP6 and CYP9 subfamilies as much as any other ant. The number of CYP4 and CYP6 gene families in L. niger genome is within the diversity range of other ants. Genes of the $9 \mathrm{e} 2$ family are organized in 12 duplicated clusters, with genes of $6 \mathrm{~K}$, 6A and 4G families also exhibiting tandem duplications.

\section{Positive selection in cytochrome P450 genes}

For phylogenetic analyses and selection tests we selected only complete and nearly complete ( $>250$ codons) P450 genes where the conservative heme-binding motif Phe-X-X-Gly-X-Arg-X-Cys-X-Gly was present (29 out of 40 CYP9, 5 out of 9 CYP6, and 8 out of 10 CYP4 for L. niger).

In the phylogenetic tree, most $L$. niger genes form 10 clusters near those of $\mathrm{C}$. floridanus, another ant in Formicinae subfamily (Fig. 3). Most of the CYP9 genes in the examined species are under positive selection according to pairwise $\mathrm{Z}$ test (Figs. 3 and 4, Additional file 1: Table S4). Except for recently duplicated genes phylogenetic trees of CYP4 and CYP6 always show $A$. mellifera and $N$. vitripennis as outgroups. L. niger contain two duplications of CYP4 genes after the divergence of Lasius and Camponotus lineages. Five $L$. niger genes of CYP6 family sit on the phylogenetic tree separately, but form clusters with $C$. floridanus genes. Two are putative pseudogenes. Most ant genes from the CYP4 and CYP6 families were under positive selection since their divergence with other hymenoptera according to a pairwise Z-test for selection

As noted previously, the CYP4 and CYP6 gene families are not amplified in Lasius niger. Nearly all ants with sequenced genomes have between 10 and 15 fragments of CYP4 genes, coding for more than half of the protein (including between 2 and 7 full-length genes). Camponotus has 13 full-length genes and 12 partial genes, with the partial genes coding for more than half of the protein. Non-ant hymenopteran species A.mellifera and N.vitripennis have 5 and 32 predicted CYP4 family proteins. Despite the 30 found fragments of genes, similar to the number of CYP6 found in other species, only 9 code for more than half of the protein and only 5 have the sequence of the gem-iron stabilizing motif. Other examined species have between 12 and 133 fragments of CYP6 genes, with only 6 to 32 containing coding sequences of more than $750 \mathrm{bp}$. Every ant species except for $\mathrm{C}$. obscurior has lineage specific duplication of CYP4 genes (Fig. 5). The most abundant CYP6 genes (more than 20 genes with more than half length coding region) are in invasive species (M. pharaonis, V. emeryi, L. humile, W. auropunctata, S. invicta), but in half of the genes the sequence of gem stabilizing motif is absent. The poorest representation is in C. biroi - (11 fragments total, Additional file 1: Table S4). Overall, the CYP6 tree has many species-specific duplications for all ants, except $L$. niger and $A$. cephalotes and C. obscurior (Fig. 6). The most 


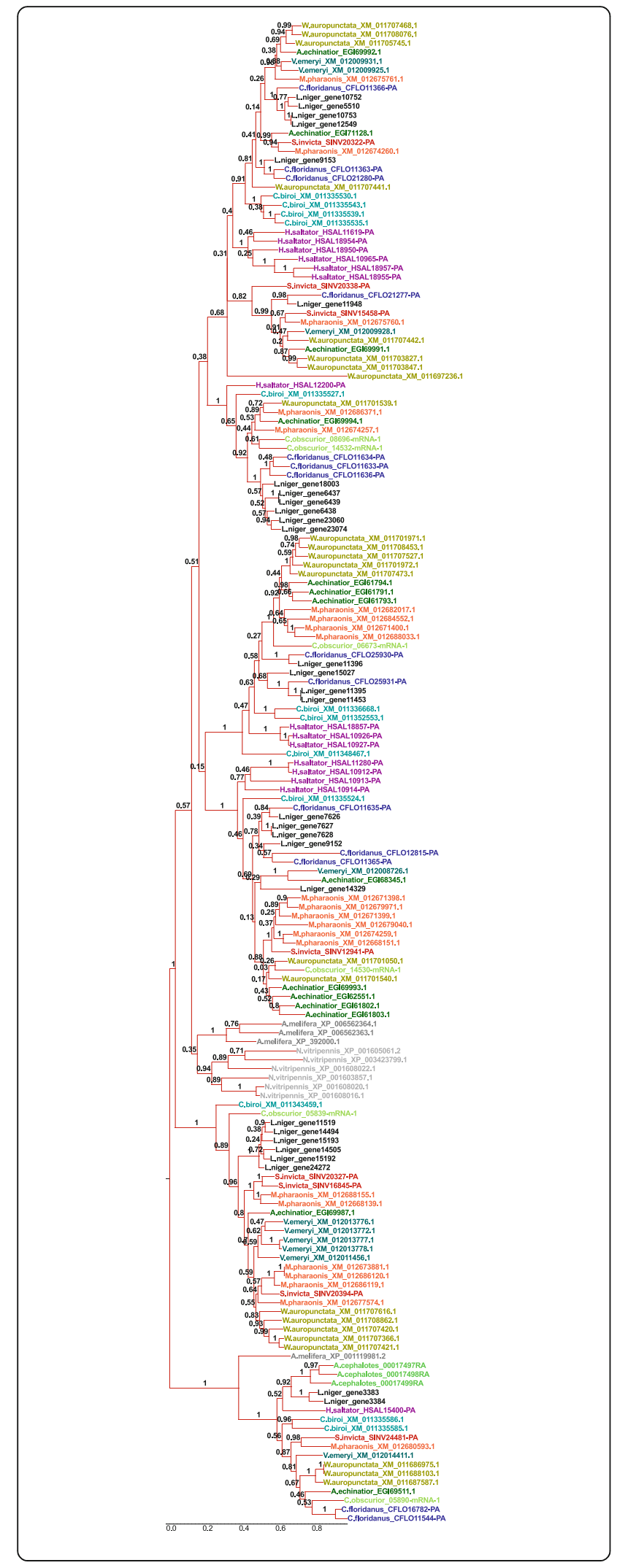

Fig. 3 Phylogenetic tree of hymenopteran CYP9 genes. Branches where statistically significant positive selection was found by Z-test are in red. Positive selection was found in most of the terminal branches of the phylogenetic tree. Many lineage-specific duplications are evident in L. niger, A. echinator and C. biroi

amplified are CYP6 in C. floridanus and S. invicta, both having 4 groups of 3-4 genes and 4 separately lying genes. Positive selection in CYP6 family, as well as in the abovementioned families, was not significant for recent duplications; most of them are in C. floridanus.

\section{Sites under selection in CYP9}

To test whether an expansion of the CYP9 family was concordant with strong evolution on gene sequences, we executed a more detailed analysis. An alignment of fulllength CYP9 coding sequences between 1425 and 1800 bp with start and stop codons was used to search for sites under positive selection. These criteria identified 3 coding CYP9 sequences in A. cephalotes, 6 sequences in A. echinator, 4 in A. mellifera, 2 in C. biroi, 11 in C. floridanus, 13 in H. saltator, 19 in L. niger, 15 in

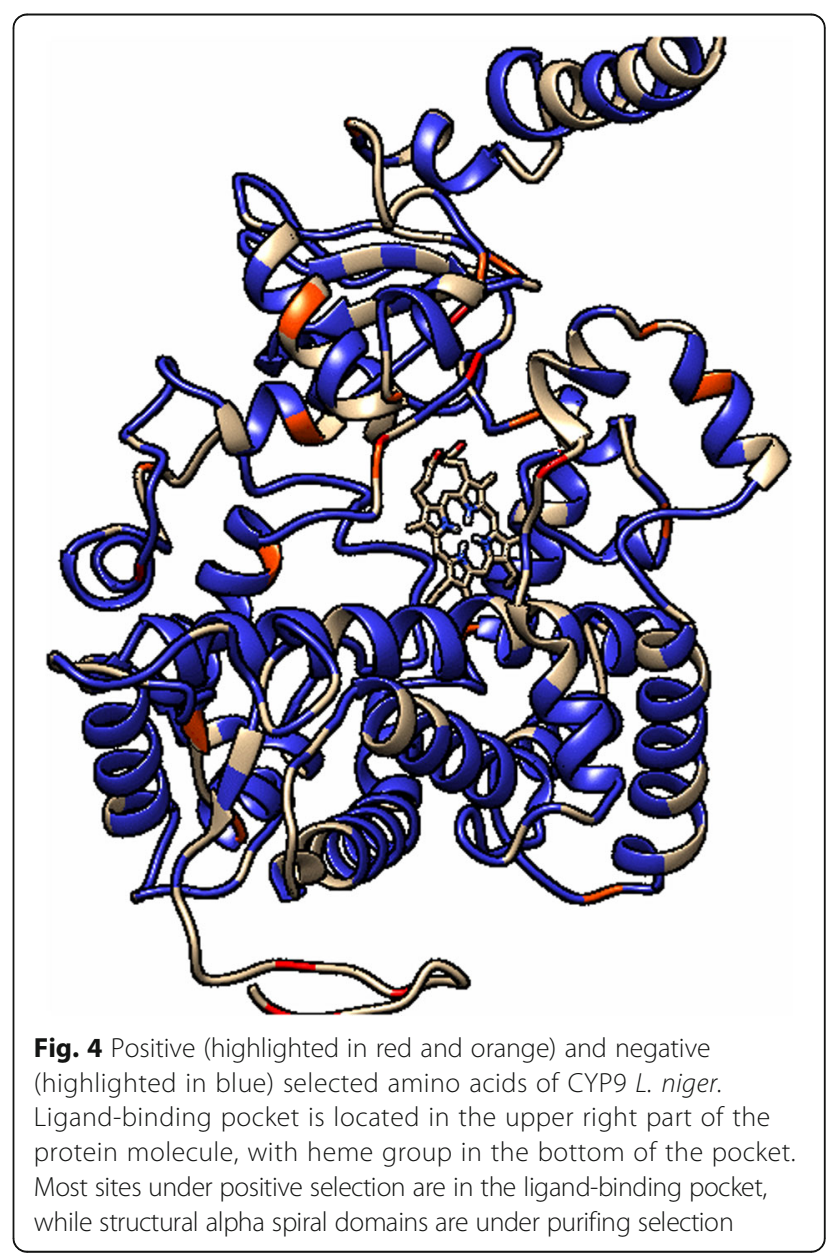




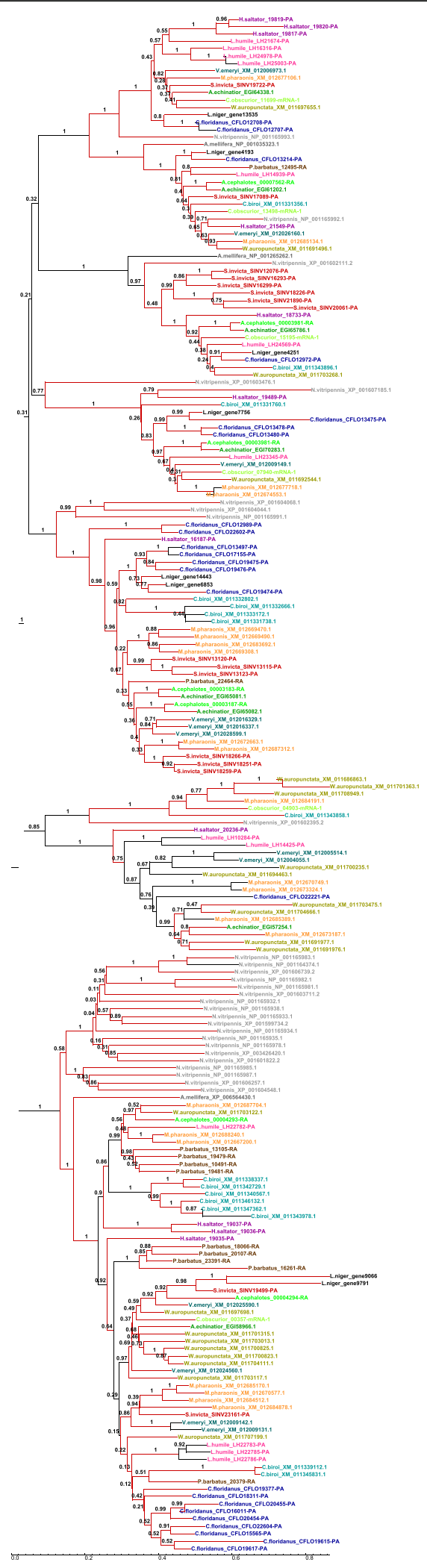

Fig. 5 Phylogenetic tree of hymenopteran CYP4 genes. Branches where statistically significant positive selection was found by Z-test are in red. Positive selection was found in most of the terminal branches of the phylogenetic tree. Many lineage-specific duplications are evident in N. vitripennis, C. floridanus and C. biroi

N. vitripennis, 8 in M.pharaonis, 5 in C.obscurior, 1 in $V$. emeryi, 3 in W.auropunctata and 5 in S. invicta.

MEME detected 59 alignment positions under positive selection $(p<0,05)$ (Additional file 1: Table S5). Three positions were confirmed by other selection search methods (SLAC and FEL, $p<0.05$, FUBAR with aposteriori probability $>0,95$ ), one was significant in all four methods. Negative selection was detected in 469 sites with statistical significance for FUBAR with aposteriori probability $>0,95$ ). Eleven positively selected positions are in ligand-binding pockets of the enzyme (Fig. 4). An additional four are at the $\mathrm{C}$-terminus, but this part of alignment was not present in all sequences. Most of negatively selected sites lie in structural domains of the enzyme.

The most frequent non-synonymous substitutions in CYP9 family did not change the charge and polarity of amino-acid residues (Val to Ile, Asp to Glu, Ile to Leu, Lys to Arg, Ser to Thr, Val to Ala) (Additional file 1: Table S6). Nevertheless, some substitutions changed charge and polarity - Lys to Glu, Asn to Asp, Ala to Thr and Leu and Ile to Met. The rarest were changes of Cys and Trp.

The MEME analysis found different preferred substitutions in different subtrees of the L.niger CYP9e tree. In one subtree, Arg was changed to Hys and Asn, while another Arg was changed to Ala. This trending of changing Arg to other amino-acids occurred only in L. niger alignments - in other ants not only Arg was changed, but also Ala and Hys. FUBAR analysis detects negative selection in most positions, where MEME detects positive. These sites are conservative in the majority of lineages but in other leading to $L$. niger diversifying selection did occur.

\section{Olfactory receptors and odorant-binding proteins}

Olfaction related genes frequently exhibited signatures of directional selection or selection relaxation. Hhmsearch and reciprocal BLASTP of $L$. niger predicted proteins revealed 75 putative odorant receptors and 3 odorant-binding proteins. One putative odorant-binding protein, having high similarity with other ant OBP, is 74 amino acids in length and is not recognized by BLASTP in the REFSEQ protein database. Other ants have additional OBPs, from 7 in $C$. floridanus and A. echinator to 20 in $V$. emeryi. Only 31 of $74 \mathrm{~L}$. niger predicted $\mathrm{OR}$ genes had coding sequence longer than $750 \mathrm{bp}$ and were used for phylogenetic analysis and selection detection. Other ants have between 118 and 474 fragments of odorant receptor genes, from 49 to 335 of this 


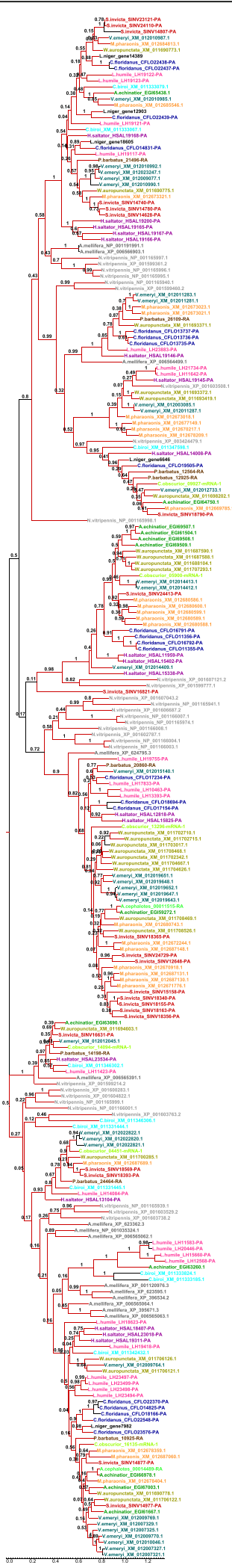

Fig. 6 Phylogenetic tree of hymenopteran CYP6 genes. Branches where statistically significant positive selection was found by Z-test are in red. Positive selection was found in most of the terminal branches of the phylogenetic tree. Many lineage-specific duplications are evident in $N$. vitripennis and $A$. mellifera

manifold were used for the phylogenetic analysis. Apis mellifera has 127 fragments of genes and 100 fragments were used to construct the phylogenetic tree. (Additional file 1: Table S7).

Genes of odorant receptors in L. niger have 5 amplified clusters -3 duplications and 25 -genes repeats. Most tandem duplications have related sequences in other ants. We did not find any $L$. niger duplication that was speciesspecific (Additional file 1: Table S8). Not only did L. niger have only 26 genes of suitable quality for odorant receptors, more than half were putative pseudogenes. Despite the poor repertoire of $L$. niger odorant receptors, half were under positive selection. In contrast to the CYP genes, odorant receptors of $L$. niger do not predominantly form clusters with $C$. floridanus genes, but often cluster with genes of more distant ant species. The phylogenetic tree of odorant receptors indicates that there are many amplifications and deletions of odorant receptor genes, providing an explantation as to why closely related A. cephalotes and A. echinator have very different odorant receptor genes, perhaps due to pseudogenisation and lineage sorting in their putative ancestor's gene set.

Z-test found positive selection in average in all sequence pairs.

\section{Fatty acid desaturases}

Cuticular hydrocarbons are important molecules in ant chemocommunication. These compounds are produced by fatty acid desaturases enzymes. Ants with sequenced genomes have from 6 in C.biroi to 14 in H. saltator. L. niger and $C$. floridanus both have 11 fatty acid desaturase genes, incomplete genes not included (Additional file 1: Table S9). Desaturases of Hymenoptera are grouped into six subfamilies, with one being unique for this order and other subfamilies orthologous to five Drosophila melanogaster desaturases (desat1, CG9743, CG9747, CG8630 and CG15331, Additional file 2).

No orthologs of D. melanogaster CG15331 were found in L. niger and P. barbatus; other ants have one. Hymenopteran specific desaturases are absent from $C$. biroi. Other subfamilies of desaturases are present in all ant genomes. In all ant species, orthologs to D.melanogaster CG9743 are under positive selection, while there was no selection within C. floridanus duplications. Almost all orthologs to D. melanogaster CG8630 and desat1 are positively selected as well. In hymenopteran specific desaturase group, the selection was significant predominantly in Myrmicinae and Formicinae subfamilies. No significant differences between $L$. niger 
and other ants can be found in desaturase gene content and selection mode (Additional file 1: Table S10).

\section{Telomerases}

Ants are known for their great intraspecific differences in life spans and $L$. niger has the greatest: from several months in males to decades in queens [22]. In contrast to Drosophila in which telomere length is maintained through retrotransposition [23] and is consistent with other studied animals species, ants' life span is based on telomerase activity. Jemielty et al. [24] showed castespecific differences in expression level of telomerases. Our annotation contains several genes related to telomerase activity. One copy of telomerase reverse transcriptase was found. Thirteen copies of another important component of telomerase ribonucleoprotein (RNP) complex - telomerase binding protein Est1a [25] - were found (this protein was also shown to play a role in nonsense-mediated mRNA decay [26]). Four genes similar to telomerase Cajal body protein were found. Future functional analysis of telomerase sequences in $L$. niger could shed light on life span regulation in this species.

\section{Vitellogenins}

Vitellogenins were duplicated in many formicoid lineages. Caste-specific expression is characteristic for vitellogenin paralogs in ants. However, the C. floridanus genome contains only one vitellogenin gene. We found only one vitellogenin in the Lasius genome, with high similarity to the Camponotus vitellogenin. HMM search for hymenopteran vitellogenins also revealed only one vitellogenin in Harpegnatos, Cerapachys and Pogonomyrmex. Other ant species had more vitellogenins; we found 3 vitellogenins in Solenopsis, 4 in Linepithema and Wasmania and 5 vitellogenins in Volenhovia and Monomorium. One more sole vitellogenin in Formicinae can not, of course, reject a hypothesis of all-Formicidae initial vitellogenin duplication, but add some more doubts to it. All mentioned vitellogenins were vgg, we did not search for vgf.

\section{Discussion}

\section{High loads of fungal parasites and retroelements}

Here, we assembled the draft genome of $L$. niger and compared it with the genomes of other ant species. Surprisingly, retrotransposons are an order of magnitude more abundant in this ant compared to others. Discovered retrotransposon genes do not contain internal stop codons and expected to be active. We also discovered the expansion of DNA repair proteins. Here, we hypothesize that expansion of DNA repair proteins could be a genome response to the expansion of retroelements. DNA repair system takes part in transposon integration and is required to restore DNA damage caused by incorrect integration.
TEs are known to interfere with host repair, therefore the diversification of DNA repair system may represent the host's response to retrotransposon expansion [27]. We further hypothesize that the diversification of DNA repair system might be an exaptation pre-adapting $L$. niger to explore urban environments. Specifically, enhanced DNA repair enzyme repertoire may play a role in resistance against DNA-damaging xenobiotics such as polycyclic aromatic hydrocarbons.

Furthermore, both protein and 18S rRNA BLAST results show high level of fungal DNA from genus Ophiocordyceps. These fungi are well-known entomopathogens [28], infecting various insects including ants. Fungal $18 \mathrm{~s}$ rDNA from L. niger assembly is most similar (95\%) to sequences from O. unilateralis infecting Camponotus carpenter ants, suggesting high rate of fungal infection in L. niger. However, characteristic fruit bodies protruding from the dead ant's head have never been seen in $L$. niger. It was shown that laboratory settings that Ophiocordyceps infects and kills many ant species besides its natural host [29] However, reproduction and spore dispersal of Ophiocordyceps requires complex host manipulation (infected ants attach to tree twigs in death grip), which is successful only in its natural host. Two species of Camponotus (C. herculeanus and C. fallax) live in the Moscow region [30] and they may be natural hosts of Ophiocordyceps found in L. niger. Alternatively, L. niger might be a natural host for this fungus but its transmission does not involve typical fruit body formation. Nevertheless, the observed fungal DNA level suggests that Ophiocordyceps infection may be an important cause of mortality among $L$. niger and there may be the selective pressure toward resistance to this pathogen.

\section{CYP9 expansion}

Insects can cope with pollution in two ways - physiological or behavioral. Metabolic detoxification of xenobiotics is an example of physiological adaptation. We studied the cytochrome P450 superfamily in detail, as it is known for its crucial role in resistance to xenobiotics [31, 32]. Compared to other ants, L. niger has a highly amplified CYP9 subfamily. Phylogenetic analysis and comparison of CYP9 genes of $L$. niger with $A$. mellifera and $N$. vitripennis allocated all genes of this subfamily in ants into subfamilies CYP9E and CYP9P. Functions of these subfamilies are unknown, but closely related subfamilies (CYP9F and CYP9G) are known for their roles in insecticide resistance [33, 34].

Entomopathogenic Cordycepitales are known to synthesize wide range of toxic compounds $[35,36]$. We have previously shown using molecular docking and virtual screening [37] that $L$. niger CYP9 cytochromes form complexes with low free energy with fusarin $C$, fumonisin, ergot-alkaloids, enniatin and beauvericin. These compounds are produced 
by Ophiocordyceps and are important pathogenicity factors [28, 36, 38]. For some of L. niger CYP9 free energy of complexes with fusarin are below $-480 \mathrm{~kJ} / \mathrm{mol}$ that suggests high affinity of enzymes to this substrate. We hypothesize, therefore, that the expansion of CYP9 in $L$. niger genome may result from coevolution with enthomopathogenic fungi.

Cytochrome P450 superfamily evolution in Formicidae was extensively studied [39]. Differential expansions and contractions of P450 subfamilies were observed in different genera. These changes are linked with adaptations to novel ecological niches and changes in colony lifestyle. Trophic specialization was found to promote the contraction of P450 family in P. barbatus [40].

L. niger and C. floridanus, which belong to the subfamily Formicinae, contain approximately the same number of cytochrome P450 genes, and this number is higher than that in other species of ants. Like L. niger, C. floridanus dwells in an urban environment and similarly omnivorous [41]. Its diet includes living and dead insects and the secretions of aphids and scale insects [42]. Cytochrome P450 genes have wide range of substrates and single amino acid substitution can drastically change substrate specificity [43]. It is possible that the large set of cytochrome P450 genes provide for polyphagia by enabling specific and nonspecific neutralization of various phyto and mycotoxins (phytotoxin resistance would enable $L$. niger to feed on nectar and resistance to mycotoxins would enable it to feed on dead insects). Cytochrome P450 genes may also be important in resistance to urban pollutants of different chemical composition (polycyclic hydrocarbons, plastics, and petrochemicals). Some cytochrome $\mathrm{P} 450$ genes can oxidize some of these chemicals, e.g., benzo(a)pyren [44].

The importance of detoxification system in L. niger xenobiotic resistance may be further increased due to scarcity of anti-fungal protective behaviour. For example, $L$. niger does not remove aphids infected with parasitic fungi from symbiotic aphid colonies, while this behaviour is common in other aphid-herding ants such as Formica s. str. (Novgorodova, in press).

\section{Odorant communication}

Genes of odorant receptors and odorant-binding proteins, including partial sequences, appear to be less abundant in the assembly of $L$. niger than in other ant genomes. We hypothesize that the Lasius genus lost some odorant receptor and odorant-binding genes in the course of their evolution.

Our reanalysis of previously published data enables unbiased comparison between species. In S. invicta, there are only 151 fragments of odorant receptors and odorantbinding proteins, instead of 297 complete genes and more than 100 fragments that were previously reported [1]. This observation could be explained by the fact that the insect odorant receptors were considered to be homologous to odorant receptors of vertebrates prior to 2013 [45]. Accordingly, G-protein coupled receptors were treated as hypothetically odor detection related, while now we know that all insect odorant receptors are ion channels. Another published prediction that was not confirmed in our work, is an increase in amounts of odorant receptors in ants as compared to A. mellifera and N. vitripennis. On the contrary, we observed that, in ants, odorant receptors and odorant-binding protein families underwent gene loss. This loss may be caused by transition of ants to terrestrial lifestyle and, therefore, increase in the role of antennal contacts in species communication (J. Reznikova, pers. Comm.). L. niger was shown to prefer visual information over pheromone trails when these stimuli were in conflict, contrary to other ant species [46, 47]. Specifically in respect to urban environments, odorant receptor loss can be very useful, as it is increasing the resistance of the species to repellents.

\section{Conclusions}

In conclusion, we hypothesize that a number of seemingly unrelated events - expansion of DNA repair, olfactory system genes loss and CYP9 gene amplification - can be viewed as preadaptations required for the successful colonization of urban areas. Among these preadaptations, the changes in olfactory system genes and CYP genes provide two different ways to fight against xenobiotics. The latter is achieved rather by avoiding poisonous chemicals versus metabolizing them with the help of detoxication system, thus, opening the potential for trade-off between these two systems. Moreover, this hypothesis is experimentally supported [48] by a negative correlation between CYP and odorant-binding proteins expression levels in insects. In our future work, we will test whether urban-life associated selection sweeps are enriched in the genes that comprise xenobiotic avoidance and metabolizing systems.

\section{Additional files}

Additional file 1: Tables S1-13. Samples, number of CYP, OR, OBP and desturases genes, results of selection detection, neuropeptides. (XLSX $47 \mathrm{~kb}$ )

Additional file 2: Phylogenetic tree of ants fatty acid desaturases. 1 hymenoptera-specific desaturases, 2 D. melanogaster CG9747 orthologs, 3 - D. melanogaster CG9743 orthologs, 4 - D. melanogaster desat1 orthologs, 5 - D. melanogaster CG8630 orthologs, 6 - D. melanogaster CG15331 orthologs. (PDF 2930 kb)

Acknowledgments

Authors thank N. Yu. Oparina and T. S. Putyatina for useful discussion.

Declarations

This article has been published as part of BMC Evolutionary Biology Vol 17 Suppl 1, 2017: Selected articles from BGRSISB-2016: evolutionary biology. The full contents of the supplement are available online at https:/bmcevolbiol.biomedcentral.com/ articles/supplements/volume-17-supplement-1. 


\section{Funding}

This work was supported by the Russian Scientific Foundation, project no. 14-14-00330 - specimen collecting, DNA extraction, library preparation, sequencing, genome assembly and publication fee and project no. 16-1600007 - data analysis and discussion.

\section{Availability of data and materials}

The genome assembly and predicted protein sequences have been deposited in the GenBank under the BioProject ID PRJNA171386.

\section{Authors' contributions}

VAS conceived and designed the experiment, EAK and SNL collected the animal samples, extracted DNA and RNA and prepared Illumina libraries, all authors participated in sequence data analysis, EAK, MAN, SVN and VAS wrote the manuscript. All authors read and approved the final manuscript.

\section{Competing interests}

The authors declare that they have no competing interests.

\section{Consent for publication}

Not applicable.

\section{Ethics approval and consent to participate}

The animal use protocol was performed in accordance with Bioethics Committee of Lomonosov Moscow State University. At present, the committee do not provide a specific approval numbers for invertebrate animal research. Animals used in this study do not belong to regulated or endangered species.

\section{Author details}

${ }^{1}$ Faculty of Biology, Department of Evolution, Lomonosov Moscow State University, Moscow, Russian Federation. ${ }^{2}$ Faculty of Bioengineering and Bioinformatics, Lomonosov Moscow State University, Moscow, Russian Federation. ${ }^{3}$ Belozersky Institute for Physicochemical Biology, Lomonosov Moscow State University, Moscow, Russian Federation. ${ }^{4}$ Molecular and Computational Biology, University of Southern California, Los Angeles, CA 90089, USA. ${ }^{5}$ Department of Applied Mathematics \& Mathematical Biology and Bioinformatics Laboratory, St.Petersburg State Polytechnical University, St.Petersburg 195251, Russia.

\section{Published: 7 February 2017}

\section{References}

1. Wurm Y, Wang J, Riba-Grognuz O, Corona M, Nygaard S, Hunt BG, .. Dijkstra MB. The genome of the fire ant Solenopsis invicta. Proc Natl Acad Sci. 2011;108(14): 5679-5684.

2. Smith CD, Zimin A, Holt C, Abouheif $E_{1}$ Benton $R_{1}$ Cash $E_{1} \ldots$ \& Fave MJ. Draft genome of the globally widespread and invasive Argentine ant (Linepithema humile). Proc Natl Acad Sci. 2011a;108(14):5673-5678.

3. Oxley PR, Ji L, Fetter-Pruneda I, McKenzie SK, Li C, Hu H, ... \& Kronauer DJ. The genome of the clonal raider ant Cerapachys biroi. Curr Biol. 2014;24(4):451-458.

4. Bonasio R, Zhang G, Ye C, Mutti NS, Fang X, Qin N, ... \& Zhang P. Genomic comparison of the ants Camponotus floridanus and Harpegnathos saltator. Science. 2010;329(5995):1068-1071.

5. Seifert B. Lasius platythorax n. sp., a widespread sibling species of Lasius niger (Hymenoptera: Formicidae). Entomologia Generalis. 1991;16(1):69-81.

6. Fjerdingstad EJ, Gertsch PJ, Keller L. The relationship between multiple mating by queens, within-colony genetic variability and fitness in the ant Lasius niger. J Evol Biol. 2003;16(5):844-85.

7. Fjerdingstad EJ, Keller L. Relationships between phenotype, mating behavior, and fitness of queens in the ant Lasius niger. Evolution. 2004;58(5):1056-63.

8. Putiatina TS. Effect of recreational pressure on ant communities of open biocenoses in Moscow. Mosc Univ Biol Sci Bull. 2011;66(1):42-5.

9. Ewing B, Green P. Base-calling of automated sequencer traces using phred. II. Error probabilities. Genome Res. 1998;8(3):186-94.

10. Bolger AM, Lohse M, Usadel B. Trimmomatic: a flexible trimmer for Illumina sequence data. Bioinformatics. 2014:30(15):2114-2120.

11. Bankevich A, Nurk S, Antipov D, Gurevich AA, Dvorkin M, Kulikov AS, ... \& Pyshkin AV. SPAdes: a new genome assembly algorithm and its applications to single-cell sequencing. J Comput Biol. 2012;19(5):455-477.

12. Parra G, Bradnam K, Ning Z, Keane T, Korf I. Assessing the gene space in draft genomes. Nucleic Acids Res. 2009;37(1):289-97.
13. Simão FA, Waterhouse RM, loannidis P, Kriventseva EV, Zdobnov EM. BUSCO: assessing genome assembly and annotation completeness with single-copy orthologs. Bioinformatics. 2015;31:3210-2.

14. Stanke M, Steinkamp R, Waack S, Morgenstern B. AUGUSTUS: a web server for gene finding in eukaryotes. Nucleic Acids Res. 2004;32 suppl 2:W309-12.

15. Conesa A, Götz S, Garcia-Gomez JM, Terol J, Talon M, Robles M. Blast2GO: a universal tool for annotation, visualization and analysis in functional genomics research. Bioinformatics. 2005;21:3674-6.

16. Schwarz W. 40 puzzles and problems in probability and mathematical statistics. New York: Springer Science \& Business Media, LLC; 2007.

17. Nei $M$, Gojobori T. Simple methods for estimating the numbers of synonymous and nonsynonymous nucleotide substitutions. Mol Biol Evol. 1986;3(5):418-26.

18. Smit AFA, Hubley R, Green P. RepeatMasker Open-4.0. 2013-2015. http://www. repeatmasker.org. Accessed 7 Oct 2016.

19. Weidong B, Kenji K. Kojima, Oleksiy Kohany Repbase Update, a database of repetitive elements in eukaryotic genomes. Mobile DNA. 2015;6:11.

20. Grimaldi D, Agosti D. A formicine in New Jersey Cretaceous amber (Hymenoptera: Formicidae) and early evolution of the ants. Proc Natl Acad Sci. 2000;97(25):13678-83.

21. Werck-Reichhart D, Feyereisen R. Cytochromes P450: a success story. Genome Biol. 2000;1(6):3003.

22. Kutter H, Stumper R. Hermann Appel, ein leidgeadelter entomologe (1892-1966). Proceedings of the Sixth International Congress of the International Union for The Study of Social Insects (Bern); 1969. pp. 275-279.

23. Mason JM, Biessmann H. The unusual telomeres of Drosophila. Trends Genet. 1995;11(2):58-62.

24. Jemielity S, Kimura M, Parker KM, Parker JD, Cao X, Aviv A, Keller L. Short telomeres in short-lived males: what are the molecular and evolutionary causes? Aging Cell. 2007;6(2):225-33.

25. Reichenbach $P$, Höss M, Azzalin CM, Nabholz M, Bucher P, Lingner J. A human homolog of yeast Est1 associates with telomerase and uncaps chromosome ends when overexpressed. Curr Biol. 2003;13(7):568-74.

26. Huntzinger E, Kashima I, Fauser M, Saulière J, Izaurralde E. SMG6 is the catalytic endonuclease that cleaves mRNAs containing nonsense codons in metazoan. RNA. 2008;14(12):2609-17.

27. Baydoun HH, Pancewicz J, Nicot C. Human T-lymphotropic type 1 virus p30 inhibits homologous recombination and favors unfaithful DNA repair. Blood. 2011:117(22):5897-906.

28. De Bekker C, Quevillon LE, Smith PB, et al. Species-specific ant brain manipulation by a specialized fungal parasite. BMC Evol Biol. 2014;14:166. doi:10.1186/s12862-014-0166-3.

29. Wang XL, Yao YJ. Host insect species of Ophiocordyceps sinensis: a review. ZooKeys. 2011;127:43.

30. Czechowski W, Radchenko A, Czechowska W, Vepsäläinen K. The ants of Poland with reference to the myrmecofauna of Europe. Warszawa: Natura optima dux Foundation; 2012.

31. Hlavica P. Insect cytochromes P450: topology of structural elements predicted to govern catalytic versatility. J Inorg Biochem. 2011;105(10):1354-64.

32. Itokawa K, Komagata $\mathrm{O}$, Kasai $\mathrm{S}$, Kawada H, Mwatele C, Dida GO, ... \& Tomita T. Global spread and genetic variants of the two CYP9M10 haplotype forms associated with insecticide resistance in Culex quinquefasciatus Say. Heredity. 2013;111(3):216-226

33. Zhao SS, Zhao GD, Di TY, Ding H, Wan XL, Li B, ... \& Wei ZG. Analyzing the promoters of two CYP9A genes in the silkworm Bombyx mori by dualluciferase reporter assay. Molecular biology reports. 2013:40(2):1701-1710.

34. Sandstrom P, Welch WH, Blomquist GJ, Tittiger C. Functional expression of a bark beetle cytochrome P450 that hydroxylates myrcene to ipsdienol. Insect Biochem Mol Biol. 2006;36(11):835-45.

35. Zheng P, Xia Y, Xiao G, Xiong C, Hu X, Zhang S, ... \& Zhao GP. Genome sequence of the insect pathogenic fungus Cordyceps militaris, a valued traditional Chinese medicine. Genome Biol. 2011;12(11):R116.

36. Wichadakul D, Kobmoo N, Ingsriswang S, Tangphatsornruang S, Chantasingh D, Luangsa-Ard JJ, Eurwilaichitr L. Insights from the genome of Ophiocordyceps polyrhachis-furcata to pathogenicity and host specificity in insect fungi. BMC Genomics. 2015;16(1):1.

37. Konorov EA, Nikitin MA. Amplification of CYP9 genes as a preadaptation of the black garden ant Lasius niger to urban conditions. Mol Biol. 2015;49(3):403-7.

38. Araújo JPM, Evans HC, Geiser DM, Mackay WP, Hughes DP. Unravelling the diversity behind the Ophiocordyceps unilateralis (Ophiocordycipitaceae) complex: Three new species of zombie-ant fungi from the Brazilian Amazon. Phytotaxa. 2015;220(3):224-38. 
39. Simola DF, Wissler L, Donahue G, Waterhouse RM, Helmkampf M, Roux J, ... \& Reese JT. Social insect genomes exhibit dramatic evolution in gene composition and regulation while preserving regulatory features linked to sociality. Genome Res. 2013;23(8):1235-1247.

40. Smith CR, Smith CD, Robertson HM, Helmkampf M, Zimin A, Yandell M, Holt $\mathrm{C}, \mathrm{Hu} \mathrm{H}$, Abouheif E, Benton R, et al. Draft genome of the red harvester ant Pogonomyrmex barbatus. Proc Natl Acad Sci. 2011 b;108:5667-5672.

41. Ferster B, Deyrup M, Scheffrahn RH, Cabrera BJ. The pest ants of Florida. Gainesville, FL: Instit. Food Agric. Sci; 2000.

42. Field Guide to Urban Wildlife. Common Animals of Cities \& Suburbs How They Adapt \& Thrive. Mechanicsburg: Feinstein Julie. Stackpole Books; 2011. ISBN-10: 0811705854

43. Wen Z, Baudry J, Berenbaum MR, Schuler MA. Ile115Leu mutation in the SRS1 region of an insect cytochrome P450 (CYP6B1) compromises substrate turnover via changes in a predicted product release channel. Protein Eng Des Sel. 2005;18(4):191-9.

44. Indra R, Moserova M, Sulc M, Frei E, Stiborova M. Oxidation of carcinogenic benzo [a] pyrene by human and rat cytochrome P450 $1 \mathrm{~A} 1$ and its influencing by cytochrome b5-a comparative study. Neuro Endocrinol Lett. 2013;34 Suppl 2:55-63.

45. Liu YC, Pearce MW, Honda T, Johnson TK, Charlu S, Sharma KR, ... \& Dahanukar A. The Drosophila melanogaster phospholipid flippase dATP8B is required for odorant receptor function. PLoS Genet. 2014;10:e1004209.

46. Grüter C, Czaczkes TJ, Ratnieks FLW. Decision making in ant foragers (Lasius niger) facing conflicting private and social information. Behav Ecol Sociobiol. 2011:65:141. doi:10.1007/s00265-010-1020-2.

47. Aron $\mathrm{S}$, Beckers R, Deneubourg JL, et al. Memory and chemical communication in the orientation of two mass-recruiting ant speciesIns. Soc. 1993;40:369. doi:10.1007/BF01253900.

48. Bautista MAM, Bhandary B, Wijeratne AJ, Michel AP, Hoy CW, Mittapalli O. Evidence for trade-offs in detoxification and chemosensation. 2015.

\section{Submit your next manuscript to BioMed Central and we will help you at every step:}

- We accept pre-submission inquiries

- Our selector tool helps you to find the most relevant journal

- We provide round the clock customer support

- Convenient online submission

- Thorough peer review

- Inclusion in PubMed and all major indexing services

- Maximum visibility for your research

Submit your manuscript at www.biomedcentral.com/submit

C) Biomed Central 